\title{
The tooth on-a-chip: a microphysiologic model system mimicking the pulp-dentin interface and its interaction with biomaterials
}

Cristiane Miranda França ${ }^{1 *}$, Anthony Tahayeri ${ }^{1^{*}}$, Nara Sousa Rodrigues ${ }^{2}$, Shirin Ferdosian $^{1}$, Regina Puppin-Rontani ${ }^{3}$, Jack L. Ferracane ${ }^{1}$, Luiz E. Bertassoni ${ }^{1,4,5,6}$

1 Department of Restorative Dentistry, School of Dentistry, Oregon Health \& Science University, Portland, OR, USA.

2 Post-Graduation Program in Dentistry, Federal University of Ceará, Fortaleza, Ceará, Brazil.

3 School of Dentistry, University of Campinas, Piracicaba, Sao Paulo, Brazil

4 Center for Regenerative Medicine, School of Medicine, Oregon Health \& Science University, Portland, OR, USA.

5 Department of Biomedical Engineering, School of Medicine, Oregon Health \& Science University, Portland, OR, USA.

6 Cancer Early Detection Advanced Research Center (CEDAR), Knight Cancer Institute, Portland, OR, USA

* These authors contributed equally to this work.

\section{Abstract}

The tooth has a unique configuration with respect to biomaterials that are used for its treatment. Cells inside of the dental pulp interface indirectly with biomaterials via a calcified permeable membrane, formed by a dentin barrier which is composed of several thousands of dentinal tubules ( $2 \mu \mathrm{m}$ in diameter) connecting the dental pulp tissue to the outer surface of the tooth. Although the cytotoxic response of the dental pulp to biomaterials has been extensively studied, there is a shortage of in vitro model systems that mimic the dentin-pulp interface, enabling an improved understanding of the morphologic, metabolic and functional influence of biomaterials on live dental pulp cells. To address this shortage, here we developed an organ-ona-chip model system which integrates cells cultured directly on a dentin wall within a microdevice which replicates some of the architecture and dynamics of the dentinpulp interface. The tooth-on-a-chip is made out of molded polydimethylsiloxane (PDMS) with a design consisting of two chambers separated by a dentin fragment. To characterize pulp cell responses to dental materials on-chip, stem cell-derived odontoblasts were seeded onto the dentin surface, and observed using live-cell microscopy. Standard dental materials used clinically (2-hydroxyethylmethacrylate HEMA, Phosphoric Acid - PA, and Adper-Scotchbond - SB) were tested for 
cytotoxicity, cell morphology and metabolic activity on-chip, and compared against standardized off-chip controls. All dental materials had cytotoxic effects in both onchip and off-chip systems in the following order: HEMA $>S B>P A(p<0.05)$, and cells presented consistently higher metabolic activity on-chip than off-chip $(p<0.05)$. Furthermore, the tooth-on-a-chip enabled real-time tracking of odontoblast monolayer formation, remodeling, and death in response to biomaterial treatments, and gelatinolytic activity in a model hybrid layer $(\mathrm{HL})$ formed in the microdevice. In conclusion, the tooth-on-a-chip is a novel platform that replicates near-physiologic conditions of the pulp-dentin interface, and enables live-cell imaging to study dental pulp cell response to biomaterials.

Keywords: stem cells; bioengineering; MMPs; biocompatibility; restorative materials; odontoblast

\section{Introduction}

Treatment of dental diseases, such as caries or dentin hypersensitivity, require the application of a biomaterial directly onto a cavity formed on the tooth surface. Typically, these involve the attachment of the biomaterial onto dentin - the calcified tissue underlying the outer dental enamel. Therefore, the structural configuration of the tooth results in the formation of an unique interface, where biomaterials contact the dentin matrix, and indirectly allow reaction byproducts and leachates to diffuse into the underlaying dental pulp. Such interactions are enabled by the presence of dentinal tubules ( $2 \mu \mathrm{m}$ in diameter), which are distributed across the dentin matrix, and house odontoblast cell processes that extend a significant length into the dentinal tubules. Therefore, together with the dental pulp, a restored tooth forms an intricate biomaterials/dentin/cell complex (Bertassoni 2017; Bertassoni et al. 2012) that is unique in the body. The biocompatibility and cytotoxicity of dental materials on pulp cells have been extensively studied. Existing model systems include cells cultured on plates (Caldas et al. 2019; Chaves et al. 2012; Schmalz and Galler 2017), larger devices that require specialized equipment, such as the Hume model 
(Hume 1984), the in-vitro pulp chamber (Hanks et al. 1988), and the dentin barrier test (Schmalz et al. 1999), or ex-vivo models such as the rodent slice culture (Murray et al. 2000), and entire human tooth culture (Camilleri et al. 2014). Despite their extensive usefulness, these models have limited ability for direct observation (i.e. live cell imaging) of the morphologic and metabolic events that occur as cells inside of the tooth become exposed to biomaterials overtime. Arguably, these events hold important information regarding the ability of the tooth to respond to different biomaterials and treatments.

Organs-on-a-chip, integrate microengineered substrates with microfluidics technologies to replicate levels of tissue functionality that are difficult to achieve with conventional $2 \mathrm{D}$ or $3 \mathrm{D}$ cell culture systems. Similarly, these miniaturized organ systems allow for straightforward experimental control over multifactorial questions that are too difficult to systematically study in-vivo (Bhatia and Ingber 2014; Esch et al. 2015). Several organ-on-a-chip models have demonstrated outstanding ability to replicate the complex multicellular architecture, cell-cell/cell-matrix interactions, tissue mechanics, that are naturally present in complex tissues (Huh et al. 2010; Kim et al. 2012; Torisawa et al. 2014). For instance, a recent gut on-chip-model was engineered to replicate the human gut epithelial microvilli, vasculature, microflora, and peristalsis (Kim et al. 2012). Similar methods have also been used to engineer the liver-on-a-chip (Prot et al. 2012), lung-on-a-chip (Huh et al. 2012), bone-marrowon-a-chip (Torisawa et al. 2014), and several others (Bhatia and Ingber 2014; Esch et al. 2015). Despite these extensive examples, this technology has remained largely underdeveloped in the scope of dental research, and thus the development of an onchip model of the tooth has remained elusive. 
Here we report the development, optimization, and testing of a miniaturized model of the pulp-dentin interface, which we refer to as the 'tooth-on-a-chip'. We determine the real-time response of pulp-cells to various dental materials such as phosphoric acid, adhesive monomers, and dental adhesives. We then compare the cytotoxicity, metabolic activity, and morphologic changes of an engineered pulp tissue interfaced with the native human dentin as exposed to these materials in comparison to an ISO in-vitro model. We show that the tooth-on-a-chip provides direct visualization of the complexity of the pulp-dentin-biomaterials interface, and enables real-time assessment of the response of pulp cells to dental materials on a level that was was not possible.

\section{Materials and Methods}

\section{Microdevice fabrication}

A master mold was created from a $1 \mathrm{~mm}$-thick sheet of poly-methyl methacrylate (PMMA) (Figure 1A) using a laser cutter. The PMMA molds were used to make an impression on a transparent layer of polydimethylsiloxane (PDMS) (Fig 1B, C, D) which was fabricated with 4 reservoirs for cell medium. Human dentin from third molars extracted for orthodontic reasons according to the institutional ethics committee guidelines was used. Teeth were sectioned into fragments of $500 \mu \mathrm{m}$ in thickness, inserted into the PDMS device (Figure 1E), and positioned on a plasmatreated coverslip (Figure 1F). The fully assembled microdevice replicates the interface of dentin with the dental pulp on one side and the dental material with dentin on the other, thus forming two accessible chambers representing the "pulp 
side" and the "cavity side", respectively (Figure 1G, H). For details, see Supplementary information.

A

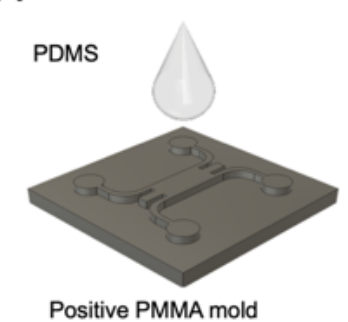

E

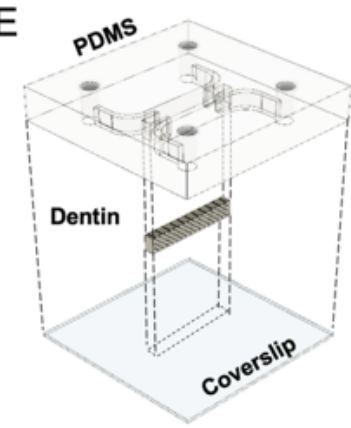

B

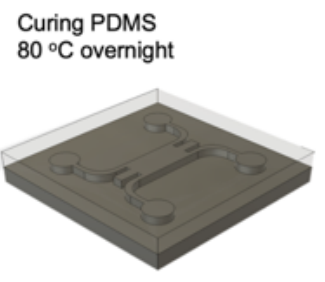

$\mathrm{F}$

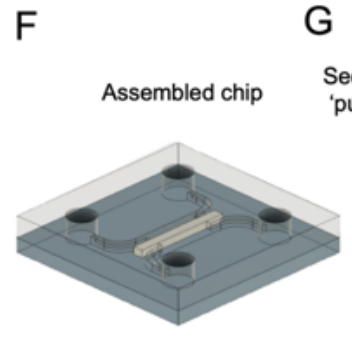

G
C

Removal of cured PDMS
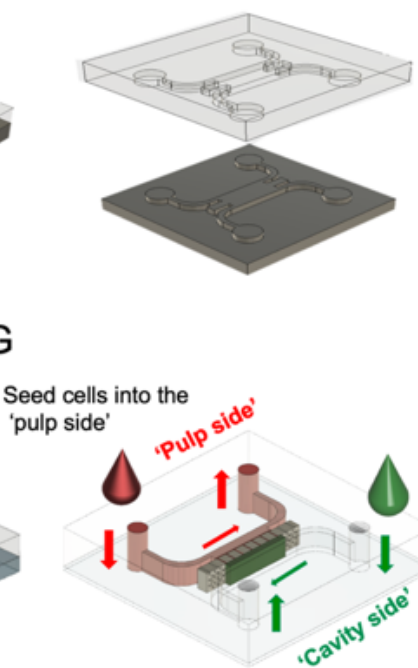

Figure 1: Fabrication of the tooth-on-a-chip. (A) PDMS prepolymer is poured onto a positive PMMA mold, and (B) cured overnight at $80^{\circ} \mathrm{C}$. Next, (C) the PDMS is released from the template and (D) $8 \mathrm{~mm}$ holes are punched at the end of the channels to form reservoirs. (E) The PDMS and coverslips are plasma-treated, and a dentin fragment is placed in the center, between the two chambers and $(F)$ the system is assembled. Two different chambers representing the 'pulp side' and the 'cavity side' are formed. (G) The assembled microdevice with dentin as a semipermeable membrane is shown in $(H)$.

\section{Cell culture}

Stem cells from apical papilla (SCAPs) were cultured for 10 days in odontogenic medium, as detailed in the supplementary information. A suspension of $20 \mu \mathrm{L}$ with $10^{5} \mathrm{SCAP} / \mathrm{ml}$ was seeded into the 'pulp side' and incubated for $1 \mathrm{~h}$ to promote cell contact and attachment onto the dentin wall. Next, (Figure $1 \mathrm{H}$ ) reservoirs were filled with $100 \mu \mathrm{L}$ of cell medium. Cells were cultured for 7 days with daily cell medium changes.

\section{Live-cell imaging}


To demonstrate that the tooth-on-a-chip enables live-cell imaging of the cells in close contact with the dentin, a monolayer of odontogenically differentiated SCAP cells was imaged overnight, every 30 minutes using a spinning disk confocal microscope (Supplementary information). For the cytotoxicity experiments, on days 1 and 7, chips $(n=4)$ were fixed with $4 \%$ paraformaldehyde, stained for actin filaments and nuclei, and imaged using a confocal microscope (LSM 880 Zeiss) (Supplementary information). The whole monolayer in contact with dentin was photographed in 3 consecutive images and analyzed using ImageJ (Fiji, NIH, Maryland, USA).

\section{Cytotoxicity}

After the monolayer formation, three dental materials were tested: (a) HEMA dissolved in cell culture medium at a known cytotoxic concentration of $10 \mathrm{mM}$, (b) 37\% phosphoric acid gel (PA) (Ultradent Products, South Jordan, UT, USA) used to etch the dentin on-chip for 15s, and (c) 35\% PA plus Adper Single Bond 2 (SB) (3M/ESPE, St Paul, MN, USA) applied per manufacturer recommendations (Supplementary information). The materials were introduced into the 'cavity side' of the dentin, thus forming an interface similar to the dentin-pulp complex in a restored tooth $(n=4)$. Live-cell images of SCAPs assembling as a monolayer on the dentin surface were obtained using a spinning disk field scanning confocal microscope (Yokogawa CSU-X1, Japan). To identify dead cells, SCAPs were incubated with $50 \mathrm{nM}$ of Helix NP NIR (Biolegend, San Diego, CA), a DNA-binding dye, diluted in cell culture medium 10 minutes before imaging. This dye does not require rinsing, so cells continued to be imaged to provide a baseline. After three minutes of imaging, $20 \mathrm{mM}$ of HEMA was added to the opposite side of the dentin and livecell images were taken every 10 minutes for one hour. 
We then compared on-chip experiments against experiments performed using the ISO-10993-1 (ISO 2009), which we refer to as the off-chip group. To that end, $10^{4}$ SCAPs/well were seeded in 96-well plates and after $24 \mathrm{~h}$, cells were supplemented with the ISO recommended concentrations of HEMA, PA, and extracts of SB obtained by immersing the photopolymerized SB disks in culture media $(n=6)$ (Supplementary information). Next, cells were incubated for $24 \mathrm{~h}$ with the conditioned medium of each dental material, and the conditioned medium was replaced with the untreated medium for 7 days. Controls consisted of samples cultured in standard culture medium not exposed to the dental materials. Cell metabolic activity was measured using Alamar Blue (ThermoFisher) on days $0,1,3$, 5 , and 7 .

\section{On-chip zymography of hybrid layer degradation}

After determining the cytotoxicity of HEMA, PA, and SB, we evaluated the contribution of MMPs released by dental pulp cells in the degradation of the hybrid layer $(\mathrm{HL})$ formed after SB treatment. We hypothesized that the immediate response of pulp cells to acid attack and monomer exposure might stimulate cells to secrete substantial amounts of proteases, which may stimulate HL degradation in-vivo - a phenomenon that cannot be measured using traditional off-chip zymography methods without cells. To that end, we applied SB as previously described to the 'cavity side' of chips fabricated with or without SCAPs $(n=4)$. On-chip zymography of HL degradation was performed as detailed in supplementary information using fluorescein-conjugated gelatin (DQ ${ }^{T M}$ gelatin, EnzChek Gelatinase Assay Kit, ThermoFisher) in the cavity and pulp sides of the device incubated for at least $48 \mathrm{~h}$, 
after which the proteolytic activity was imaged via confocal microscopy.

\section{Statistics}

Results were analyzed using either a Student's t-test, one-way ANOVA or two-way ANOVA followed by Tukey post-hoc tests $(\alpha=0.05)$ on GraphPad Prism 8.

\section{Results}

Morphogenesis of SCAP on dentin-pulp interface on-a-chip

The tooth-on-a-chip was fabricated with a $500 \mu \mathrm{m}$ thick dentin barrier and a monolayer of differentiated SCAPs on the opposite site of the dentin wall to emulate a deep cavity.

Figure 2 A-D shows a time lapse of the monolayer assembly on the dentin wall (Figure 2 A) until the complete attachment to the dentin is visible (Figure 2 D). We also tracked the cell death by incubating SCAPs with Helix NP NIR (Biolegend, San Diego, CA), a fluorescent dye that only binds to the DNA of non-viable cells (Figure 2 E-H). Next, we added $20 \mathrm{mM}$ of 2-hydroxyethyl methacrylate (HEMA) (Esstech, PA, USA) to the 'cavity side' of the chip and images were taken every 10 minutes. When cells died, the nuclei emitted fluorescence on the $500-530 \mathrm{~nm}$ wavelength. Only cells located up to $60 \mu \mathrm{m}$ away from the dentin were considered as part of the odontoblast-like monolayer. 


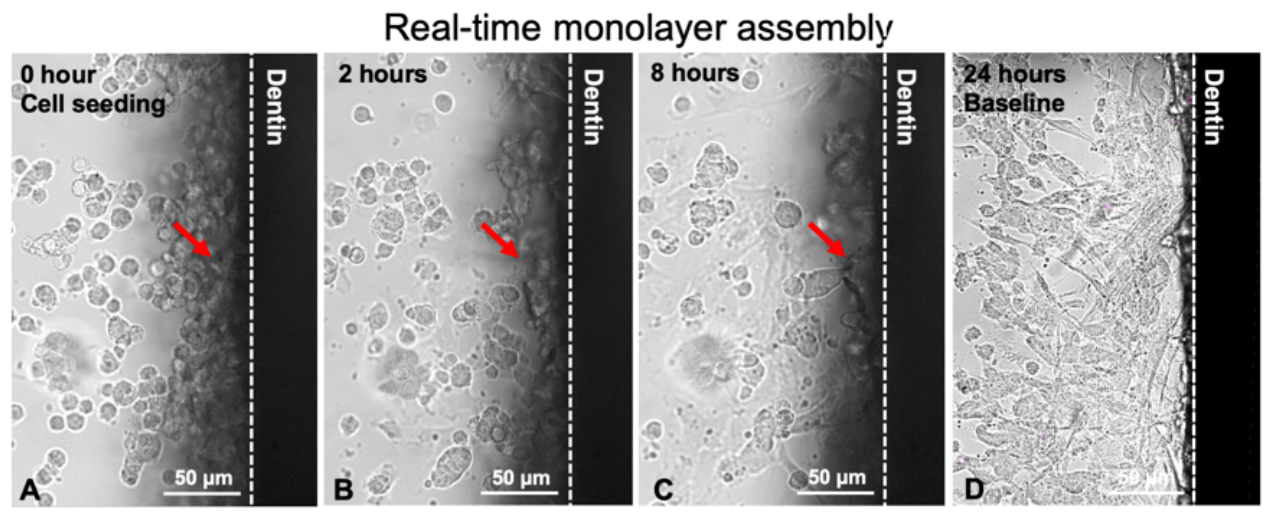

Real-time cytotoxic effect of 20 mM HEMA

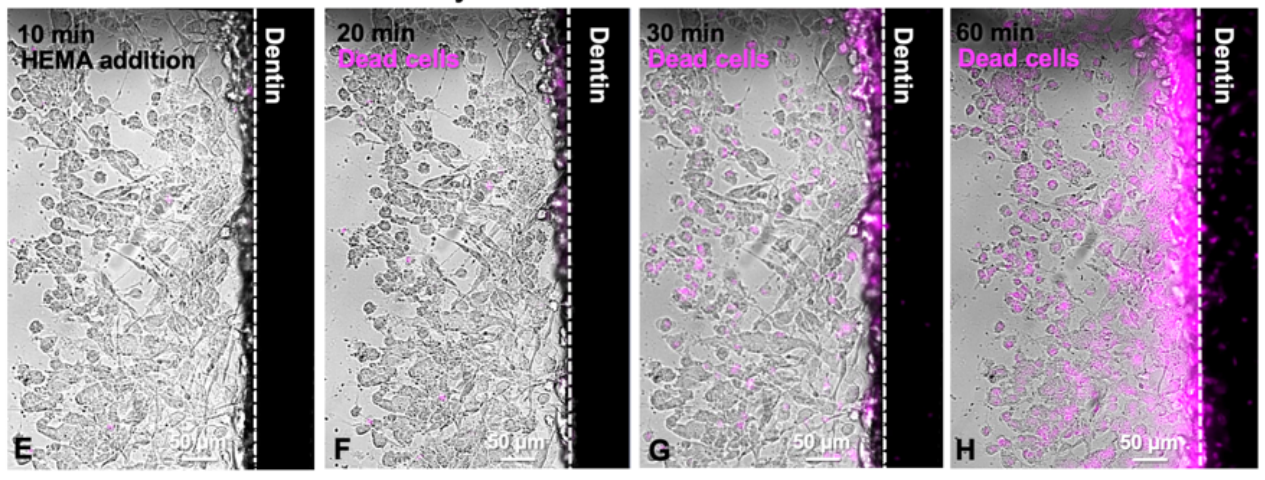

Figure 2: Live-cell imaging on-chip. $10^{5}$ stem cells from apical papilla were seeded on-chip (A) showing that almost $50 \%$ of cells were spread in 4 hours (B) and complete cell spreading was observed in 8 hours (C). After 12 hours the monolayer was completely formed (D). Arrows show the morphological changes of a single cell, which goes from being round and unattached, to a spread morphology as it attaches to the dentin wall after 8 hours (A-D, arrow). Cells cultured on-chip after 24 hours were incubated with a DNA dye (Helix NP NIR) and imaged, demonstrating initial cell viability near $100 \%$ (E). Next, 20 mM HEMA was added to the 'cavity side' of the chip and after 10 minutes of incubation cells still showed high viability (F). After 30 minutes, almost $50 \%$ of cells had their nuclei stained, which is suggestive of high cell death (G). After 60 minutes, nearly all cells were not viable $(\mathrm{H})$. (Confocal microscopy, $647 \mathrm{~nm}$ and bright field, dye - Helix NP NIR). Still images extracted from movies 1 and 2 in supplementary material.

While the effects of HEMA were visible on-chip, we also sought to demonstrate the feasibility of real time observation of cell response to biomaterials while the material was being applied. To that end, figure 3 shows a sequence of images of the cell monolayer on-chip as the dentin fragment is exposed to phosphoric acid, following the recommended clinical steps and time of acid application on the tooth. The interaction of the dentin with the acid results in the formation of a number of microbubbles, which appear to stem from the dentin tubules and intertubular dentin matrix (Figure 3 A-D). Interestingly, a discrete contraction of the cell monolayer 
bioRxiv preprint doi: https://doi.org/10.1101/748053; this version posted August 28, 2019. The copyright holder for this preprint (which was not certified by peer review) is the author/funder, who has granted bioRxiv a license to display the preprint in perpetuity. It is made available under aCC-BY-NC-ND 4.0 International license.

towards the dentin wall is also seen, as if the cells appeared to contract in response to the acid at the interface with the tooth (Figure 3 E-L).
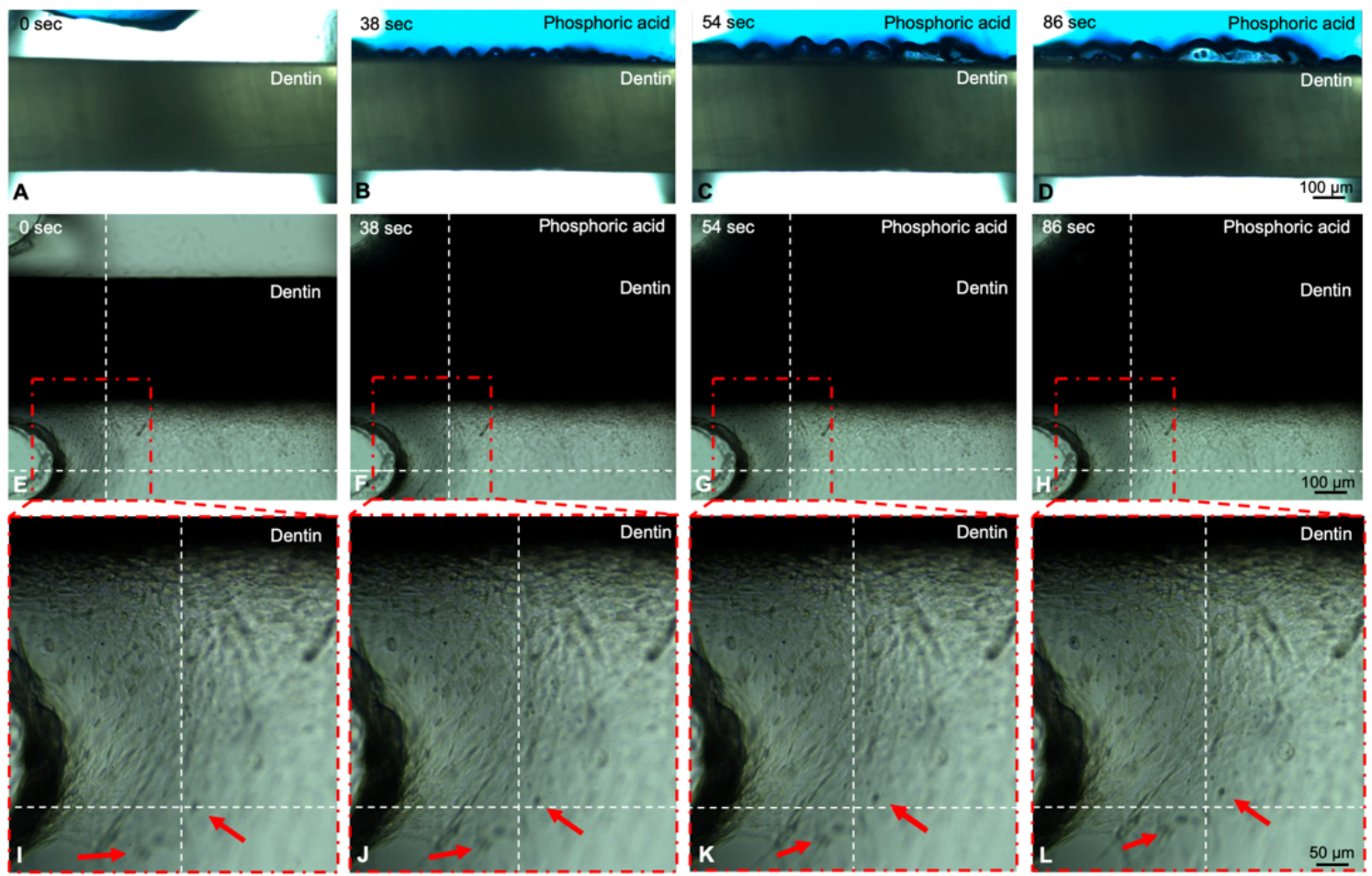

Figure 3: Time lapse showing the process of dentin acid etching with $35 \%$ phosphoric acid. Figures A-D show live imaging of the acid reacting with dentin (cells are not seen due to the intensity of light). Figures E-L present the same process with low light intensity to demonstrate the cell monolayer as the dentin is being acid etched. Figures I-L show what appears to the be the contraction of cells in the monolayer as a function of exposure to the phosphoric acid. Red arrows show bundles of cells that appear to move relative to the dotted line, which is provided as reference. Using the scalebar as reference, it is visible that cells appear to move approximately $50 \mu \mathrm{m}$ over 86 seconds. The supplementary movies (movies 3 and 4 ) of these events show a clear contraction of the monolayer as a whole.

\section{Effect of dental materials on SCAP morphology and proliferation}

Each tested material elicited apparent cellular injury as early as $24 \mathrm{~h}$ after treatment for both on-chip (Figure 4 A-D) and off-chip (Figure 4 E-H) samples. The monolayer in the HEMA group consisted of poorly connected round cells with pyknotic nuclei

(Figure 4 B) while off-chip HEMA was significantly more cytotoxic, presenting a 10- 
fold reduction in cell number $(p<0.05)$ relative to the untreated controls (Figure $4 \mathbf{F}$, I). PA etching of the dentin on-chip caused more discrete monolayer disorganization, cytoplasmic injuries, and separation of cells from the dentin fragment (Figure 4C). Similar cytoplasmic changes were seen in off-chip samples, although overall the effect appeared to be far more significant than off-chip. SB treatment off-chip also caused cytoplasmic changes characterized by a dim actin stain and increased intercellular spaces (Figure 4 D), with a visible decrease in cell number. After 7 days, there were striking differences between on-chip (Figure 4 K-M) and off-chip (Figure 4 O-Q) samples regarding cell number and morphology, except for the untreated control samples (Figure $4 \mathbf{J}, \mathbf{N}$ ). Quantitatively, cells numbers were significantly higher on-chip compared to off-chip controls after 7 days for all treatments, with both HEMA and SB leading to a significant reduction in cell number relative to untreated controls on day 1 (Figure 4 I-R) $(p<0.05)$.
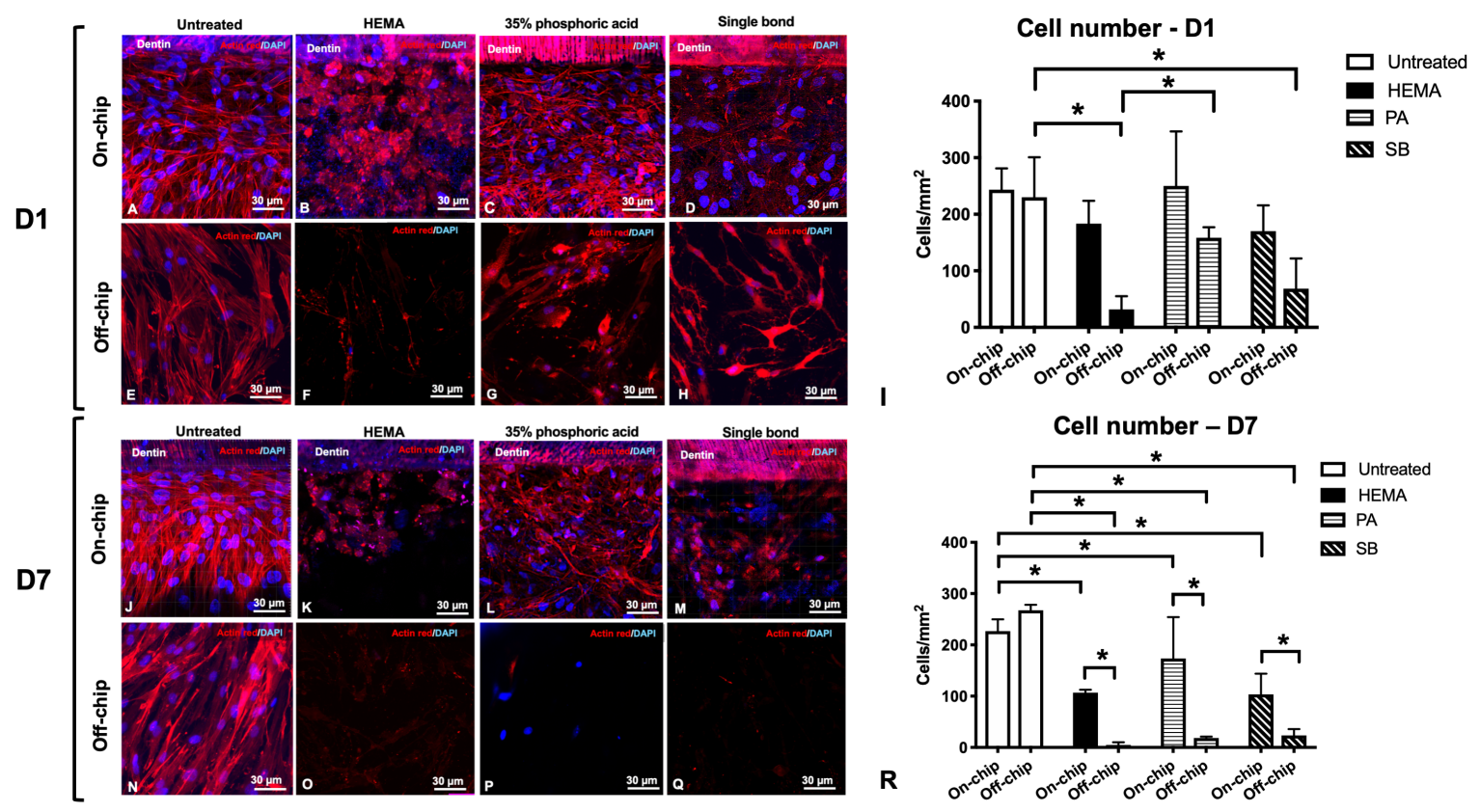

Figure 4: Cytotoxicity of SCAPs on-chip and off-chip. On day 1 untreated samples (A) had SCAP monolayers that were morphologically stable for at least 7 days, while HEMA, PA and SB groups (B, $C$, and $D$ respectively) showed significant cell morphology changes and decreased cell number onchip. Cells cultured off-chip on day 1 showed polygonal morphology with oval nuclei in the control group (E), and almost no cells were visible after HEMA treatment. Severe cytoplasmic changes and apparently fragmented nuclei where seen for PA $(G)$ and SB groups $(H)$. Cells on-chip on day 7 
showed consistent monolayers on untreated and phosphoric acid samples $(\mathrm{J}, \mathrm{L})$ while HEMA and SB groups $(K, M)$ had signs of severe cell degradation. $(\mathrm{N})$ Untreated groups off-chip showed confluent cell monolayers, whereas HEMA, PA, and $\mathrm{SB}(\mathrm{O}, \mathrm{P}, \mathrm{Q}$ respectively) displayed very few cells and with faint cytoplasms. Cell count in the monolayer for day 1 and 7 indicated more cells on-chip than offchip after dental materials application. (Two-way ANOVA, $\left.{ }^{*} p<0.05\right)$

\section{Metabolic activity}

Cells cultured on-chip showed consistently higher metabolic activity than cells cultured off-chip (Figure 5). On-chip, both untreated samples as well as samples treated with PA did not present a significant decrease in metabolic activity over time (Figure 5A, C). Samples treated with HEMA and SB, on the other hand, had a significantly lower metabolic activity only after 7 days $(p<0.05)$. Off-chip, untreated cells presented a spontaneous decrease in cell metabolism as shortly as $24 \mathrm{~h}$ (Figure 5A), with HEMA, PA, and SB showing a significant and continuous decrease in metabolic activity from day 1 to day 7 (Figure 5B-D) $(p<0.05)$.

A

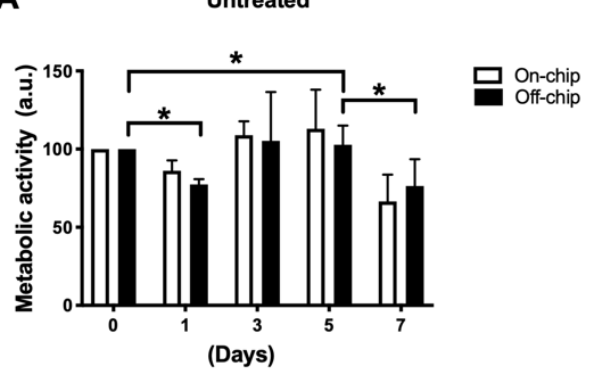

C

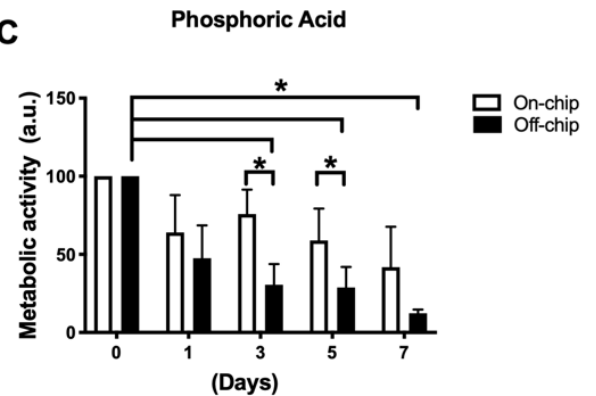

B

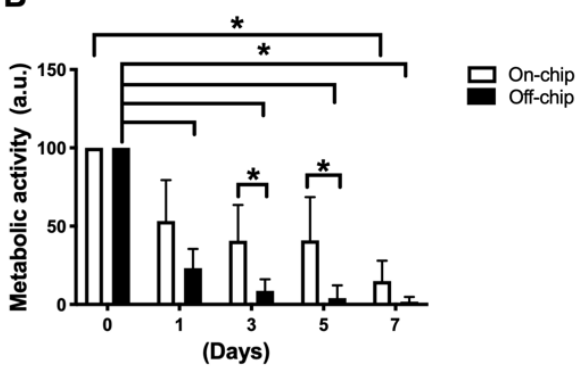

D

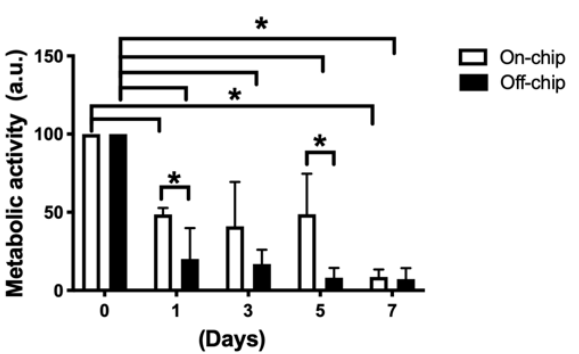

Figure 5: Comparison of metabolic activity between cultures on-chip and off-chip. Cells cultured on-chip had higher metabolic activity than cells cultured off-chip using ISO standards. HEMA (B) was the most cytotoxic material followed by SB (D) and PA (C). (Two-way ANOVA, * $p<0.05$ ) 


\section{In-situ zymography on-chip}

To determine the contribution of cellular gelatinases to $\mathrm{HL}$ degradation, in situ zymography of the HL was performed with fluorescein-conjugated gelatin on chips cultured with or without cells. Green fluorescence, indicative of MMP activity, was visible after $24 \mathrm{~h}$ and peaked within $48 \mathrm{~h}$ for both groups (Figure 6). Chips seeded with cells had visibly greater HL fluorescence than chips incubated without cells (Figure 6A, F). Interestingly, an intense green fluorescence was detected in colocalization with cell cytoplasm, indicating that the conjugated gelatin was also hydrolyzed at the monolayer (Figure $6 \mathbf{H}, \mathbf{J}$ ). Conversely, chips without cells presented discrete MMP activity at the HL and inside dentin tubules close to the adhesive (Figure 6G).

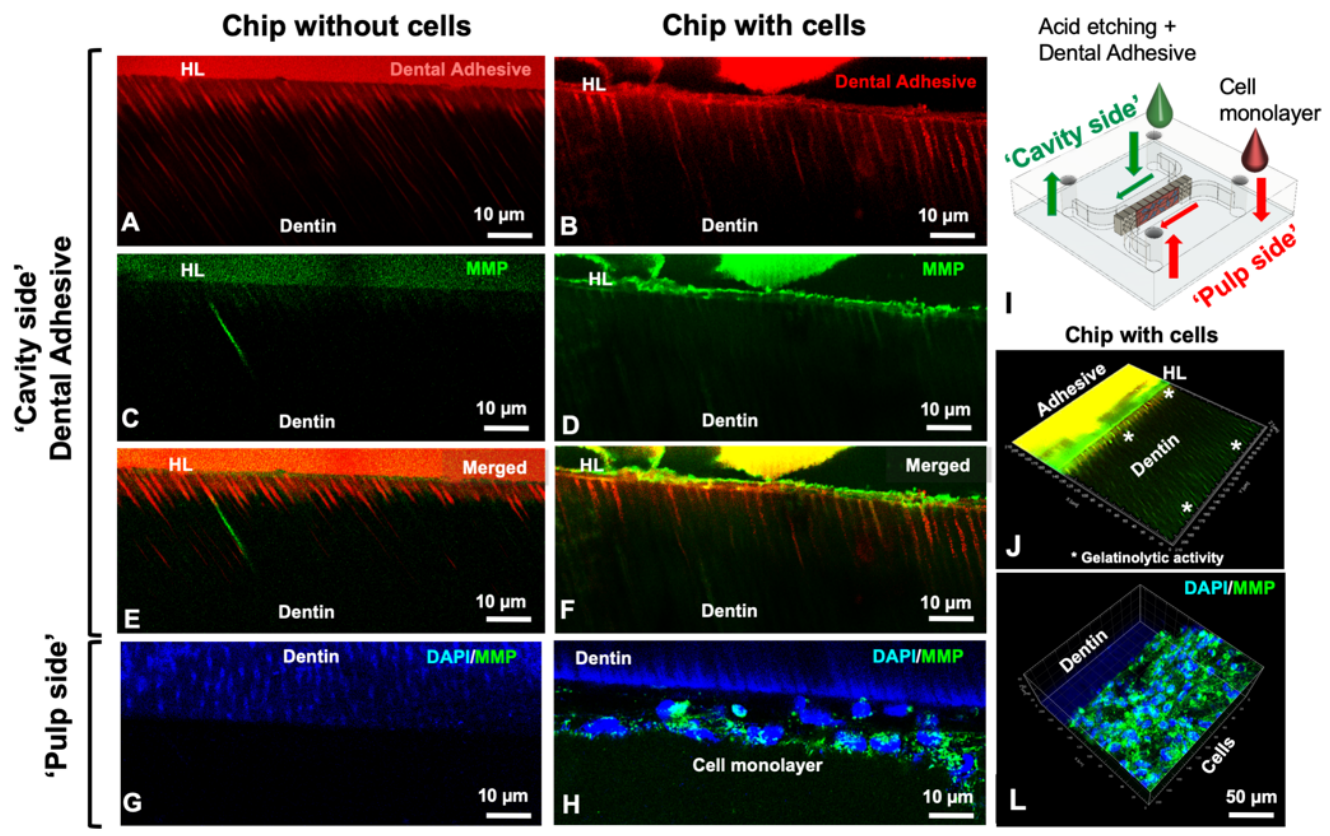

Figure 6: Gelatinolytic activity in the hybrid layer on-chip with and without cells after $48 \mathbf{~ h}$. Hybrid layer and tags present in chips without (A) and with cells (B). Fluorescein-conjugated gelatin showing gelatinolytic activity in the $\mathrm{HL}$ and dentin tubules (C-F). No evidence of gelatinolytic activity on the 'pulp side' for chips without cells $(G)$ while for chips with cells, gelatinolytic activity was colocalized with cell cytoplasm $(\mathrm{H})$. Schematic of the chip (I) and 3D orthogonal view of the adhesive side of a chip with cells $(\mathrm{J})$ showing gelatinolytic activity in the hybrid layer inside dental tubules $\left(^{*}\right)$ and on the cell side $(L)$, unquenched gelatin co-localized with cell cytoplasm. 


\section{Discussion}

Current understanding of the biological interactions of restorative materials stems from in-vitro experiments using material-based approaches that cannot replicate the complexity of the true biological phenomena occurring at the pulp-dentin interface. Improved model systems that enable direct visualization and quantification of the biological events occurring at the pulp-dentin interface when subjected to different dental materials would address these limitations. To that end, the tooth-on-a-chip provides a highly controllable 3D environment that emulates the pulp-dentin interface in-vivo, allowing for investigation of human pulp cell responses in real-time and in the context in which these interactions occur - that is, at the interface of the dental material with the dentin, forming a permeable barrier to the pulp cells.

One of the key prerequisites for mimicking the dental pulp in-vitro is the establishment of a stable cell-dentin interface comprised of an odontoblast-like cell monolayer that interfaces with the dentin wall and responds to stimuli. First, we tried to encapsulate the cells in a hydrogel to promote a 3D environment on-chip; however, cells did not form monolayers, given that the gels prevent homogenous cell-matrix interactions at the cell-tissue interface. Therefore, we seeded high cell densities on-chip observing complete monolayer formation within 24 hours. Early cell attachment to the dentin was promoted via EDTA pre-treatment, and pre-culturing SCAPs in odontogenic medium for 7-10 days before seeding the cells on-chip. This allowed us to emulate the dentin-pulp interface by fabricating consistent monolayers in a short period of time (Figure 2 A-D), which allowed for easier reproducibility of our experiments and a larger number of replicates. We chose SCAPs because they 
have a high proliferation rate, potential to differentiate into odontogenic lineage (Miller et al. 2018; Zhang et al. 2015), and to form a typical dentin-pulp like complex in-vivo (Sonoyama et al. 2006). With the tooth-on-a-chip experiments, the combination of odontogenic medium and the dentin matrix provided cells with a microenvironment comprised of biochemical signaling, dentin nanotopography, and stiffness that is favorable to produce monolayers that are stable for several days.

We tested commonly used dental materials on-a-chip and compared their cytotoxic response against well-established ISO controls. Dental materials can interact with the dentin-pulp complex both mechanically, chemically, and indirectly via leachates that travel through the dentin tubules and diffuse through the porosity of the intertubular matrix. Therefore, for comprehensive cytotoxicity screenings, ISO determined various approaches of structuring the material-cell interface, such as direct cell contact, extract test, diffusion tests, dentin barrier and tooth slice models (ISO 2009; ISO/ADA 2008; Schmalz and Galler 2017). A key factor to be considered in the test choice is that dentin has a protective effect on dental pulp cells functioning as a source of growth factors (Ferracane et al. 2013; Salehi et al. 2016) and as a semipermeable barrier, limiting diffusion of leachates to pulp cells (Bertassoni 2017; Bertassoni et al. 2012; Hamid and Hume 1997). Thus, more sophisticated in-vitro models, such as in-vitro pulp chamber, dentin barrier tests, and tooth slices, are preferable (Hanks et al. 1988; ISO/ADA 2008; Schmalz et al. 1999; Schmalz et al. 2001). The tooth-on-a-chip is designed to include a dentin barrier (with a controllable thickness), while being compatible with direct visualization of biological phenomena in real-time, as well as virtually any standard analyses of molecular, metabolic and genetic function, such as PCR, immunostaining and metabolic activity assays like 
those performed here, as well as others (Bhatia and Ingber 2014). Considerable advantages of this microfluidic devices are the micro-volumes of reagents to maintain the cells in the system - each chip used 100-300 $\mu \mathrm{L}$ of cell culture medium while other systems using dentin disks use more than $1.5 \mathrm{ml}$ per sample (Hanks et al. 1988); the reduced dimensions of the chip, which enable a better use of human tooth since we could get around 10 dentin fragments from each tooth, being able to fabricate 10 chips with only one tooth, and most importantly the ability of real time assessment with extensive experimental control.

On-chip tests with HEMA induced cell death, pyknosis, and cytoplasmic shrinkage within 24 h (Figure 4B, I, R) without completely depleting the pulp-like tissue from viability and response, as it is expected clinically. Cellular responses to HEMA are characterized by an increase in reactive oxygen species, and mitochondrial damage (Bakopoulou et al. 2011; Spagnuolo et al. 2008), which corroborate our morphological findings on-chip. However, off-chip cells were even more sensitive to HEMA, showing a 10-times decrease in cell number and metabolic activity. Potential explanations for this finding, in addition to the lack of dentin barrier function, are that cells cultured off-chip lack the cell-matrix contact with dentin making them more susceptible to external injuries (Schmalz and Galler 2017).

Etching dentin with $35 \%$ PA for 15 seconds proved to be harmful to the cell monolayer on-chip, but not enough to fully disrupt it in $24 \mathrm{~h}$. Moreover, after 7 days the monolayer was almost completely reconstituted (Figure 4). Again, this is more consistent with clinical outcomes, where acid-etching elicits the solubilization of dentin matrix components that are important for pulp regeneration (Athirasala et al. 
2017; Ferracane et al. 2013; Salehi et al. 2016) instead of causing odontoblast death. Conversely, off-chip experiments showed that the same $15 \mathrm{~s}$ of acid application (as recommended by ISO), elicited $90 \%$ of cell death after 7 days (Figure 4) and a decrease in metabolic activity (Figure 5), indicating that cells off-chip were far more sensitive to acidic conditions. It seems that results obtained off-chip overestimated PA cytotoxicity.

Additionally, our results suggest that in $24 \mathrm{~h}$ the monolayer is partially disrupted, showing visible cytoplasmic changes (Figure 4), while on day 7 the monolayer had disappeared and cell number had decreased to $20-30 \mathrm{cell} / \mathrm{mm}^{2}$, indicating the potential toxic effect of leachates over time. Likewise, the on-chip morphological results denote different cellular cytotoxicity mechanisms for HEMA and Single Bond, partially explained by the fact that even though HEMA is a significant component of the Single Bond adhesive composition, HEMA is a freely soluble monomer with a fast action, while Single Bond adhesive is a monomer blend that was photopolymerized, thus releasing potentially toxic leachates much more slowly over time. Cell morphology changes as a consequence of these physical characteristics of the biomaterials, and in the context of the pulp-dentin interface, have remained elusive so far. Additionally, it is possible that other monomers in the composition of Single Bond i.e., UDMA and Bis-GMA may be less soluble than HEMA, in which case they diffuse more slowly (or not at all) through the dentin tubules to affect the cells (Bertassoni 2017; Bertassoni et al. 2012). This same finding could not be reproduced off-chip, and on day 1 , Single Bond application proved to be highly cytotoxic to cells, leading to nuclei changes and more than $90 \%$ of cell death on day 7. 
Lastly, we performed a functional assay with the tooth-on-a-chip, by investigating the role of cellular MMPs in hybrid layer degradation. The release of endogenous dentin MMPs that are bound to collagen fibrils in mineralized dentin and become exposed after acid etching procedures has been implicated in the $\mathrm{HL}$ enzymatic degradation, eventually deteriorating the resin-dentin bonding interface (Breschi et al. 2010; Mazzoni et al. 2012; Tjaderhane et al. 2013). Studies conducted with quenched fluorescein-conjugated gelatin in dentin slices or dentin extracts show gelatinolytic activity in the hybrid layer (Breschi et al. 2010; Gu et al. 2018; Mazzoni et al. 2012). However, the cellular role in MMP production has been less explored. In 2009, Lehmann et al. provided strong evidence that self-etching adhesives can stimulate odontoblasts to produce MMP2 (Lehmann et al. 2009). Our system is uniquely capable of culturing cells, and investigating gelatinolytic activity while simultaneously imaging the hybrid layer as formed by adhesive systems. This enables one to assess the collective effect of proteases in HL degradation, including both cell- and matrixderived proteases, which is more meaningful to the proteolytic activity occurring invivo. Here, we fabricated hybrid layers in devices with and without cells, simulating a clinical dental protocol, with all the intermediate steps observed in a clinical setting. Chips with cells presented more gelatinolytic activity in the HL, inside the intratubular dentin, and co-localized with the cell cytoplasm (Figure 6), suggesting that cells actively participate in the degradation of the $\mathrm{HL}$.

Organs-on-a-chip are bioengineering devices that attempt to reconstruct key functions of tissues and organs that cannot be modeled using other existing cell culture systems. The main challenge of an organ-on-chip is to recapitulate in vivo physiology of at least a subset of functions and then to progressively add additional 
functions over time to speed up the development of new materials, drugs, and disease models (Musah et al. 2017). Similarly to what has been done with other organ-on-a-chip models (Kim et al. 2012; Kim et al. 2016), the tooth on-a-chip opens a wide range of systematic investigations of the dentin-pulp tissue. One of the novelties of the chip is the possibility to treat the dentin using the same protocols used in the dental clinic and to image live-cell morphological changes concomitantly. The time lapses of monolayer formation and disassembling due to HEMA solubilization through dentin tubules, as well as the dentin acid etching and hybrid layer formation, demonstrate the tooth-on-a-chip as a window to track pulp cell cellular and subcellular responses in an environment more consistent with the in-vivo conditions. One limitation of this study, however, is that it is not feasible to cover all aspects of the microfluidic device at once> Future iterations of the device, which are currently being developed, include the addition of controllable flow, built-in biosensors and variable designs for different materials systems. Additionally, key biological functionality will require the addition of functional capillaries, immune cells, and innervation on the 'pulp side', and microbiome and salivary flow on the 'cavity side'. This forms the basis for future studies.

\section{Author Contribution}

CMF and AT contributed equally to this work, carried out the experiments, and analyzed the data. NSR, SF, RPR contributed to the experiments and data analyses. CMF, AT, JLF, and LEB wrote the manuscript. LEB conceived the paper, the chip design, and supervised the project.

\section{Acknowledgment}


We acknowledge Dr. Anibal Diogenes (University of Texas) for the donation of SCAPs. We acknowledge expert technical assistance from Dr. Crystal Chaw in the Advanced Light Microscopy Core at the Jungers Center at Oregon Health \& Science University. This project was supported by funding from the National Institute of Dental and Craniofacial Research (R01DE026170 and 3R01DE026170-03S1 to LEB), the Oregon Clinical \& Translational Research Institute (OCTRI) - Biomedical Innovation Program (BIP), the Innovation in Oral Care Awards sponsored by GlaxoSmithKline (GSK), International Association for Dental Research (IADR), the Michigan-Pittsburgh-Wyss Resource Center - Regenerative Medicine Resource Center (MPW-RM), the OHSU Fellowship for Diversity and Inclusion in Research (OHSU-OFDIR to CMF).

The authors declare no potential conflicts of interest with respect to the authorship and/or publication of this article. 


\section{References}

Athirasala A, Lins F, Tahayeri A, Hinds M, Smith AJ, Sedgley C, Ferracane J, Bertassoni LE. 2017. A novel strategy to engineer pre-vascularized full-length dental pulp-like tissue constructs. Sci Rep. 7(1):3323.

Bakopoulou A, Leyhausen G, Volk J, Tsiftsoglou A, Garefis P, Koidis P, Geurtsen W. 2011. Effects of hema and tedgma on the in vitro odontogenic differentiation potential of human pulp stem/progenitor cells derived from deciduous teeth. Dent Mater. 27(6):608-617.

Bertassoni LE. 2017. Dentin on the nanoscale: Hierarchical organization, mechanical behavior and bioinspired engineering. Dent Mater. 33(6):637-649.

Bertassoni LE, Orgel JP, Antipova O, Swain MV. 2012. The dentin organic matrix limitations of restorative dentistry hidden on the nanometer scale. Acta Biomater. 8(7):2419-2433.

Bhatia SN, Ingber DE. 2014. Microfluidic organs-on-chips. Nat Biotechnol. 32(8):760772.

Breschi L, Martin P, Mazzoni A, Nato F, Carrilho M, Tjaderhane L, Visintini E, Cadenaro M, Tay FR, De Stefano Dorigo E et al. 2010. Use of a specific mmp-inhibitor (galardin) for preservation of hybrid layer. Dent Mater. 26(6):571-578.

Caldas IP, Alves GG, Barbosa IB, Scelza P, de Noronha F, Scelza MZ. 2019. In vitro cytotoxicity of dental adhesives: A systematic review. Dent Mater. 35(2):195205.

Camilleri J, Laurent P, About I. 2014. Hydration of biodentine, theracal Ic, and a prototype tricalcium silicate-based dentin replacement material after pulp capping in entire tooth cultures. J Endod. 40(11):1846-1854.

Chaves CA, Machado AL, Vergani CE, de Souza RF, Giampaolo ET. 2012. Cytotoxicity of denture base and hard chairside reline materials: A systematic review. J Prosthet Dent. 107(2):114-127.

Esch EW, Bahinski A, Huh D. 2015. Organs-on-chips at the frontiers of drug discovery. Nat Rev Drug Discov. 14(4):248-260.

Ferracane JL, Cooper PR, Smith AJ. 2013. Dentin matrix component solubilization by solutions of ph relevant to self-etching dental adhesives. J Adhes Dent. 15(5):407-412.

Gu L, Mazzoni A, Gou Y, Pucci C, Breschi L, Pashley DH, Niu L, Tay FR. 2018. Zymography of hybrid layers created using extrafibrillar demineralization. J Dent Res. 97(4):409-415.

Hamid A, Hume WR. 1997. The effect of dentine thickness on diffusion of resin monomers in vitro. J Oral Rehabil. 24(1):20-25.

Hanks CT, Craig RG, Diehl ML, Pashley DH. 1988. Cytotoxicity of dental composites and other materials in a new in vitro device. J Oral Pathol. 17(8):396-403.

Huh D, Leslie DC, Matthews BD, Fraser JP, Jurek S, Hamilton GA, Thorneloe KS, McAlexander MA, Ingber DE. 2012. A human disease model of drug toxicityinduced pulmonary edema in a lung-on-a-chip microdevice. Sci Transl Med. 4(159):159ra147.

Huh D, Matthews BD, Mammoto A, Montoya-Zavala M, Hsin HY, Ingber DE. 2010. Reconstituting organ-level lung functions on a chip. Science. 328(5986):16621668.

Hume WR. 1984. An analysis of the release and the diffusion through dentin of eugenol from zinc oxide-eugenol mixtures. J Dent Res. 63(6):881-884. 
ISO. 2009. Biological evaluation of medical devices - part 5: Tests for in vitro cytotoxicity. . In: ISO, editor. International standard 10993 - biological evaluation of medical devices. 3rd Edition ed. Switzerland: ISO.

ISO/ADA. 2008. Iso 7405 - evaluation of biocompatibility of medical devices used in dentistry - modified adoption of iso 7405:2008 dentistry. In: Standardization IOf, editor. Dentistry - evaluation of bioocompatibility of medical devices used in dentistry. Geneve, Switzerland: ISO.

Kim HJ, Huh D, Hamilton G, Ingber DE. 2012. Human gut-on-a-chip inhabited by microbial flora that experiences intestinal peristalsis-like motions and flow. Lab Chip. 12(12):2165-2174.

Kim HJ, Li H, Collins JJ, Ingber DE. 2016. Contributions of microbiome and mechanical deformation to intestinal bacterial overgrowth and inflammation in a human gut-on-a-chip. Proc Natl Acad Sci U S A. 113(1):E7-15.

Lehmann N, Debret R, Romeas A, Magloire H, Degrange M, Bleicher F, Sommer P, Seux D. 2009. Self-etching increases matrix metalloproteinase expression in the dentin-pulp complex. J Dent Res. 88(1):77-82.

Mazzoni A, Nascimento FD, Carrilho M, Tersariol I, Papa V, Tjaderhane L, Di Lenarda R, Tay FR, Pashley DH, Breschi L. 2012. Mmp activity in the hybrid layer detected with in situ zymography. J Dent Res. 91(5):467-472.

Miller AA, Takimoto K, Wealleans J, Diogenes A. 2018. Effect of 3 bioceramic materials on stem cells of the apical papilla proliferation and differentiation using a dentin disk model. J Endod. 44(4):599-603.

Murray PE, Lumley PJ, Ross HF, Smith AJ. 2000. Tooth slice organ culture for cytotoxicity assessment of dental materials. Biomaterials. 21(16):1711-1721.

Musah S, Mammoto A, Ferrante TC, Jeanty SSF, Hirano-Kobayashi M, Mammoto T, Roberts K, Chung S, Novak R, Ingram M et al. 2017. Mature inducedpluripotent-stem-cell-derived human podocytes reconstitute kidney glomerular-capillary-wall function on a chip. Nat Biomed Eng. 1.

Prot JM, Bunescu A, Elena-Herrmann B, Aninat C, Snouber LC, Griscom L, Razan F, Bois FY, Legallais C, Brochot $C$ et al. 2012. Predictive toxicology using systemic biology and liver microfluidic "on chip" approaches: Application to acetaminophen injury. Toxicol Appl Pharmacol. 259(3):270-280.

Salehi S, Cooper P, Smith A, Ferracane J. 2016. Dentin matrix components extracted with phosphoric acid enhance cell proliferation and mineralization. Dent Mater. 32(3):334-342.

Schmalz G, Galler KM. 2017. Biocompatibility of biomaterials - lessons learned and considerations for the design of novel materials. Dent Mater. 33(4):382-393.

Schmalz G, Schuster U, Nuetzel K, Schweikl H. 1999. An in vitro pulp chamber with three-dimensional cell cultures. J Endod. 25(1):24-29.

Schmalz G, Schuster U, Thonemann B, Barth M, Esterbauer S. 2001. Dentin barrier test with transfected bovine pulp-derived cells. J Endod. 27(2):96-102.

Sonoyama W, Liu Y, Fang D, Yamaza T, Seo BM, Zhang C, Liu H, Gronthos S, Wang CY, Wang $S$ et al. 2006. Mesenchymal stem cell-mediated functional tooth regeneration in swine. PLoS One. 1:e79.

Spagnuolo G, D'Anto V, Valletta R, Strisciuglio C, Schmalz G, Schweikl H, Rengo S. 2008. Effect of 2-hydroxyethyl methacrylate on human pulp cell survival pathways erk and akt. J Endod. 34(6):684-688.

Tjaderhane L, Nascimento FD, Breschi L, Mazzoni A, Tersariol IL, Geraldeli S, Tezvergil-Mutluay A, Carrilho M, Carvalho RM, Tay FR et al. 2013. Strategies 
to prevent hydrolytic degradation of the hybrid layer-a review. Dent Mater. 29(10):999-1011.

Torisawa YS, Spina CS, Mammoto T, Mammoto A, Weaver JC, Tat T, Collins JJ, Ingber DE. 2014. Bone marrow-on-a-chip replicates hematopoietic niche physiology in vitro. Nat Methods. 11(6):663-669.

Zhang H, Wang J, Deng F, Huang E, Yan Z, Wang Z, Deng Y, Zhang Q, Zhang Z, Ye $J$ et al. 2015. Canonical wnt signaling acts synergistically on bmp9induced osteo/odontoblastic differentiation of stem cells of dental apical papilla (scaps). Biomaterials. 39:145-154. 


\section{Supplementary information}

\section{Fabrication of the microfluidic device}

The device design was created with a Computer Aided Design (CAD) software (Autodesk Fusion 360, Autodesk Inc, San Rafael, CA, USA) and positive template was laser cut (Boss LS1416, Boss laser, Sandorf, FL, US) in a polymethylmethacrylate (PMMA) board. Next, the templates were attached to the base of an impression container, molded with PDMS pre-polymer (Figure 1A), an oxygen-permeable, biocompatible polymer, and cured at $80^{\circ} \mathrm{C}$ overnight (Figure 1B). The set cured-PDMS negative mold was removed from the template (Figure 1C), and had four reservoirs prepared with an 8-mm punch (Figure 1D). The device is comprised of two parallel channels, two perfusable chambers (300 $\mu \mathrm{m} \mathrm{W} \times 1 \mathrm{~mm}$ $\mathrm{L} \times 1 \mathrm{~mm} \mathrm{H})$ and a central groove that holds a dentin fragment $(500 \mu \mathrm{m} \mathrm{W} \times 1 \mathrm{~mm} \mathrm{H}$ x $4.5 \mathrm{~mm} \mathrm{~L}$ cut perpendicular to the dentin tubules) (Figure $1 \mathrm{E}$ ). PDMS positive mold and coverslip were then plasma cleaned (Plasma Cleaner, PDC-32G, Harrick Plasma, Ithaca, NY, US). PDMS plasma treatment of increases silanol groups $(-\mathrm{OH})$ at the surface of the PDMS so that they form strong covalent bonds (Si- O-Si) when brought together with glass. We did not submit dentin to plasma treatment to prevent any chemical change in its structure. Thus, immediately after plasma treating PDMS mold and coverslips, a dentin fragment was carefully inserted into each PDMS mold using tweezers and the system was assembled onto the glass coverslip using slight pressure, forming a sealed and leak-proof microdevice (Figure 1 F) with two chambers separated by a semi-permeable membrane (dentin) creating distinct microenvironments for each chamber, Figure $1 \mathbf{G , H}, \mathbf{I}$. The dentin fragments had 4.5 $\mathrm{mm}$ in length, however the borders were placed within the PDMS holders (Fig 1I), 
thus the dentin area exposed to dental treatments and cell attachment in the device had about 2-2.5 $\mathrm{mm} \mathrm{L} \times 1 \mathrm{~mm} \mathrm{H}$ and $0,5 \mathrm{~mm} \mathrm{~W}$.

The microfluidic devices were then sterilized with ultraviolet light (EXFO Acticure $4000,365 \mathrm{~nm}$, at $8.5 \mathrm{~cm}$ distance, light density: $45 \mathrm{~mW} / \mathrm{cm}^{2}$ ) for $40 \mathrm{~min}$ prior to use. Each tooth on-a-chip was then filled with sterile water until use to prevent dentin dehydration.

\section{Immunofluorescence}

On day 1 and 7 , chips from each group $(n=4)$ were rinsed with phosphate-buffered saline (PBS), fixed with $4 \%$ paraformaldehyde $(\mathrm{v} / \mathrm{v})$ for $1 \mathrm{~h}$, rinsed with PBS, permeabilized with $0.1 \%(\mathrm{w} / \mathrm{v})$ Triton $\mathrm{X}-100$ for $15 \mathrm{~min}$ under agitation. Unspecific biding sites were blocked with $1.5 \%(\mathrm{w} / \mathrm{v})$ bovine serum albumin (BSA) for $1 \mathrm{~h}$. After washing with PBS, chips were incubated Actin Red 555 (cat. \# R37112, Molecular Probes, ThermoFisher) for $1 \mathrm{~h}$, rinsed with PBS and incubated with NucBlue (cat. \# R37606, Molecular Probes, ThermoFisher) for 30 min at $37^{\circ} \mathrm{C}$. Chips were imaged using a confocal microscope (Zeiss, LSM 880, Germany) with an objective of 20x (Zeiss, Plan-Apochromat 20x/0.8 M-27). The depth of imaging was 100-200 $\mu \mathrm{m}$, split into at least 20 Z-stacks. Three-dimensional (XYZ) Z-stacks were converted into TIFF files using Zen or Imaris software (v9.1, Bitplane - Oxford Instruments, Zurich, Switzerland).

\section{Cell culture}

Stem cells from apical papilla (SCAPs) (Donation from Anibal Diogenes, University of Texas) were cultured in a Minimal Essential Medium Eagle, alpha modification ( $\alpha$ MEM, Gibco, ThermoFisher Scientific, Waltham, USA) supplemented with L- 
glutamine, $100 \mathrm{U} / \mathrm{mL}$ penicillin, $100 \mu \mathrm{g} / \mathrm{mL}$ streptomycin (Sigma-Aldrich), and 10\% embryonic stem cell fetal bovine serum (eFBS, ThermoFisher). Culture media was changed every 3 days. Cells were maintained in a humidified incubator $\left(5 \% \mathrm{CO}_{2}\right.$, $37^{\circ} \mathrm{C}$ ). After reaching $80 \%$ of confluency, cells were treated with $0.05 \%$ trypsin and passaged to subsequent T75 culture plates. Only cells from passages 3 to 10 were used. Before use in experiments, cells were pre-differentiated for 7-10 days in differentiation medium (10 nM dexamethasone, $10 \mathrm{mM}$-glycerophosphate, 50 $\mu \mathrm{g} / \mathrm{mL}$ ascorbic acid) (all from Sigma-Aldrich) and 10\% eFBS.

Each chip with dentin was treated with $17 \%$ ethylenediaminetetraacetic acid (EDTA) for $45 \mathrm{~s}$ to promote cell adhesion, and rinsed thoroughly. Next, $20 \mu \mathrm{L}$ of a $10^{5}$ SCAP $\mathrm{ml}^{-1}$ media suspension was seeded into the pulp chamber and incubated vertically for $1 \mathrm{~h}\left(37^{\circ} \mathrm{C}, 100 \%\right.$ humidity, $\left.5 \% \mathrm{CO}_{2}\right)$ for cell attachment onto the dentin walls. Next, the reservoirs were filled with differentiation medium.

ISO cytotoxicity tests

To validate the chips as a microphysiologic platform to test dental materials, the following samples were used: (a) 2-hydroxyethyl methacrylate (HEMA) (cat. \# X9687044, Esstech, PA, USA) dissolved in cell culture medium (10 mM, 0.84\% v/v), (b) 37\% phosphoric acid gel (PA) (Ultradent Products Inc., South Jordan, UT, USA) dentin etching for $15 \mathrm{~s}$ and (c) 35\% phosphoric acid dentin etching followed by Adper Single Bond 2 (SB) (cat. \#51102, 3M/ESPE, St Paul, MN, USA) application according to the manufacturer recommendation. Briefly, dentin was acid-etched for $15 \mathrm{~s}$, rinsed 3 times with distilled water or until complete removal of the acid, dried with absorbent paper cone, then adhesive was applied and light-cured for 20s with a dental light (Valo Ultradent Products Inc, South Jordan, UT, USA). The materials 
were all introduced to the 'cavity side' of the dentin after, thus forming an interface akin to the dentin-pulp interface of a restored tooth $(n=4)$. We then compared onchip experiments against experiments performed using the International Organization for Standardization (ISO-10993-1) part 5 (ISO 2009). To that end, discs of Adper Single Bond 2 were prepared with $20 \mu \mathrm{L}$ of the adhesive placed inside cylindrical molds of polydimethylsiloxane (PDMS) $(4 \mathrm{~mm}$ diameter $\times 2 \mathrm{~mm}$ height) and light-cured for 10 seconds with a Valo Light (Valo Ultradent Products Inc, South Jordan, UT, USA) at a power density of $1650 \mathrm{~mW} / \mathrm{cm}^{2}$. To assure aseptic conditions, the discs were prepared inside a cell culture hood and measured with a digital caliper immediately after the cure were and then immersed in wells of a 24-well plate filled with $400 \mu \mathrm{L}$ of SCAPS culture medium for $24 \mathrm{~h}$ to keep the same weight/volume proportion of adhesive and liquid as the chip and keeping the ISO recommended range of $0.5-6.0 \mathrm{~cm}^{2} / \mathrm{mL}$ (ISO 2009; ISO/ADA 2008). After the $24 \mathrm{~h}$, the elute was filtered with a $0.22 \mu \mathrm{m}$ syringe (TPP, Darmstadt, Germany) and stored at $4{ }^{\circ} \mathrm{C}$ until use. For HEMA, cells were cultured for $24 \mathrm{~h}$ in a $10 \mathrm{mM}$ solution dissolved in cell culture medium. To prepare the phosphoric acid group, $20 \mu \mathrm{L}$ of the acid gel were dispensed onto a filter paper and immersed into $400 \mu \mathrm{L}$ of SCAP cell culture medium for $15 \mathrm{~s}$, next the elute was syringe-filtered and stored at $4{ }^{\circ} \mathrm{C}$ until use.

SCAPS were seeded in a 96-well plate $\left(10^{4}\right.$ cells/well) in $200 \mu \mathrm{L}$ of cell culture medium and after $24 \mathrm{~h}$, the culture medium was replaced with the Single Bond, HEMA and phosphoric acid extracts. Untreated cells cultured with SCAP medium served as controls. All groups had $n=6$. Cells were incubated for $24 \mathrm{~h}$ with the extracts and cell culture medium was replaced by regular SCAP medium, and cell cultures were followed for 7 days. 
Inline metabolic activity assay with Alamar Blue

Briefly, 10\% (v/v) Alamar Blue reagent (cat. \# DAL1025, ThermoFisher) was added to the medium, and chips and 96-well plates were incubated for $18 \mathrm{~h}$ to allow viable cells to convert resazurin to resorufin. Subsequently, cell medium with Alamar Blue was collected and read using a microplate reader at $570 \mathrm{~nm}$ wavelength absorbance. For the Alamar Blue controls autoclaved Alamar Blue/SCAP medium solution was used as a $100 \%$ converted control (control A) and blank Alamar Blue/SCAP medium was used as a non-converted control (control B). To calculate the reduction of viability compared to the negative control, the following equation was used:

$$
\% \text { reduction of Alamar Blue }=\frac{[(\text { sample }- \text { control } B) * 100 \%]}{\text { control } A-\text { control } B}
$$

\section{In-situ zymography of hybrid layer on-chip}

To test the hypothesis that SCAPs may contribute to production of proteases and degradation of hybrid layer $(\mathrm{HL})$ in the resin-dentin interface, we prepared the tooth on-a-chip with one group having a monolayer of SCAPs, (as described previously) and the other group with no cells. Dentin was etched for 15 s with $35 \%$ phosphoric acid gel, rinsed with continuous water irrigation, next, excess water inside the chamber was gently removed with paper cones in order to keep the cell culture medium on the opposite side of the dentin, where cells were seeded. Afterwards 7 $\mu \mathrm{L}$ of Adper Single Bond 2 adhesive labeled with $0.01 \%$ rhodamine-isothiocyanate was inserted on the etched dentin, then removed and reinserted in a back and forth movement simulating the two applications recommended in a clinical practice. After 20s the chamber with cells was protected with a photomask and the adhesive was 
photocured for 20s. $D Q^{T M}$ gelatin conjugated with fluorescein (EnzChek Gelatinase/Collagenase Assay Kit, cat \# E-12055, ThermoFisher) was reconstituted in $1 \mathrm{~mL}$ of deionized water and the chip reservoirs were filled with $320 \mu \mathrm{L}$ of cell culture medium and $80 \mu \mathrm{L}$ of $\mathrm{DQ}^{\mathrm{TM}}$ gelatin. Chips were incubated at $37 \mathrm{oC}, 5 \% \mathrm{CO} 2$ and $100 \%$ humidity. Upon proteolytic digestion, fluorescein-labelled gelatin unquenches yielding highly fluorescent peptides that were live-imaged with a confocal microscope at $48 \mathrm{~h}$.

\section{Corresponding author:}

Luiz E. Bertassoni

Biomaterials and Biomechanics, School of Dentistry

OREGON HEALTH \& SCIENCE UNIVERSITY - OHSU

2730 S.W. Moody Ave, Portland OR 97201 USA

Tel: (503) 494-8763

E-mail: bertasso@ohsu.edu 